\title{
A UNIQUE EXTREMAL METRIC FOR THE LEAST EIGENVALUE OF THE LAPLACIAN ON THE KLEIN BOTTLE
}

\author{
AHMAD EL SOUFI, HECTOR GIACOMINI AND MUSTAPHA JAZAR
}

Abstract. We prove the following conjecture recently formulated by Jakobson, Nadirashvili and Polterovich [15]: on the Klein bottle $\mathbb{K}$, the metric of revolution

$$
g_{0}=\frac{9+\left(1+8 \cos ^{2} v\right)^{2}}{1+8 \cos ^{2} v}\left(d u^{2}+\frac{d v^{2}}{1+8 \cos ^{2} v}\right),
$$

$0 \leq u<\frac{\pi}{2}, 0 \leq v<\pi$, is the unique extremal metric of the first eigenvalue of the Laplacian viewed as a functional on the space of all Riemannian metrics of given area. The proof leads us to study a Hamiltonian dynamical system which turns out to be completely integrable by quadratures.

\section{Introduction AND STATEMENT OF MAIN RESUlts}

Among all the possible Riemannian metrics on a compact differentiable manifold $M$, the most interesting ones are those which extremize a given Riemannian invariant. In particular, many recent works have been devoted to the metrics which maximize the fundamental eigenvalue $\lambda_{1}(M, g)$ of the Laplace-Beltrami operator $\Delta_{g}$ under various constraints (see, for instance, [4, 14, 16, 18, 19]). Notice that, since $\lambda_{1}(M, g)$ is not invariant under scaling $\left(\lambda_{1}(M, k g)=k^{-1} \lambda_{1}(M, g)\right)$, such constraints are necessary.

In 22, Yang and Yau proved that on any compact orientable surface $M$, the first eigenvalue $\lambda_{1}(M, g)$ is uniformly bounded over the set of Riemannian metrics of fixed area. More precisely, one has, for any Riemannian metric $g$ on $M$,

$$
\lambda_{1}(M, g) A(M, g) \leq 8 \pi(\operatorname{genus}(M)+1),
$$

where $A(M, g)$ stands for the Riemannian area of $(M, g)$ (see [7] for an improvement of this upper bound). In the non-orientable case, the following upper bound follows from Li and Yau's work [16: $\lambda_{1}(M, g) A(M, g) \leq$ $24 \pi$ (genus $(M)+1)$. On the other hand, if the dimension of $M$ is greater than 2 , then $\lambda_{1}(M, g)$ is never bounded above over the set of Riemannian metrics of fixed volume, see [5].

2000 Mathematics Subject Classification. primary : 58J50; 58E11; 35P15; secondary $37 \mathrm{C} 27$.

Key words and phrases. eigenvalue; Laplacian; Klein bottle; extremal metric, Hamiltonian system; integrable system. 
Hence, one obtains a relevant topological invariant of surfaces by setting, for any compact 2-dimensional manifold $M$,

$$
\Lambda(M)=\sup _{g} \lambda_{1}(M, g) A(M, g)=\sup _{g \in \mathcal{R}(M)} \lambda_{1}(M, g),
$$

where $\mathcal{R}(M)$ denotes the set of Riemannian metrics of area 1 on $M$.

On the other hand, in spite of the non-differentiability of the functional $g \mapsto \lambda_{1}(M, g)$ with respect to metric deformations, a natural notion of extremal (or critical) metric can be introduced. Indeed, for any smooth deformation $g_{\varepsilon}$ of a metric $g$, the function $\varepsilon \mapsto \lambda_{1}\left(M, g_{\varepsilon}\right)$ always admits left and right derivatives at $\varepsilon=0$ with

$$
\left.\frac{d}{d \varepsilon} \lambda_{1}\left(M, g_{\varepsilon}\right)\right|_{\varepsilon=0^{+}} \leq\left.\frac{d}{d \varepsilon} \lambda_{1}\left(M, g_{\varepsilon}\right)\right|_{\varepsilon=0^{-}}
$$

(see [9, 11] for details). The metric $g$ is then said to be extremal for the functional $\lambda_{1}$ under volume preserving deformations if, for any deformation $g_{\varepsilon}$ with $g_{0}=g$ and $\operatorname{vol}\left(M, g_{\varepsilon}\right)=\operatorname{vol}(M, g)$, one has

$$
\left.\frac{d}{d \varepsilon} \lambda_{1}\left(M, g_{\varepsilon}\right)\right|_{\varepsilon=0^{+}} \leq 0 \leq\left.\frac{d}{d \varepsilon} \lambda_{1}\left(M, g_{\varepsilon}\right)\right|_{\varepsilon=0^{-}} .
$$

This last condition can also be formulated as follows:

$$
\lambda_{1}\left(M, g_{\varepsilon}\right) \leq \lambda_{1}(M, g)+o(\varepsilon) \text { as } \varepsilon \rightarrow 0 .
$$

Given a compact surface $M$, the natural questions related to the functional $\lambda_{1}$ are :

(1) What are the extremal metrics on $M$ ?

(2) Is the supremum $\Lambda(M)$ achieved and, if so, by what extremal metrics?

(3) How does $\Lambda(M)$ depend on (the genus of) $M$ ?

Concerning the last question, it follows from [6] that $\Lambda(M)$ is an increasing function of the genus with a linear growth rate. Explicit answers to questions (1) and (2) are only known for the sphere $\mathbb{S}^{2}$, the real projective plane $\mathbb{R} P^{2}$ and the torus $\mathbb{T}^{2}$. Indeed, the standard metric $g_{\mathbb{S}^{2}}\left(\right.$ resp. $\left.g_{\mathbb{R} P^{2}}\right)$ is, up to a dilatation, the only extremal metric on $\mathbb{S}^{2}$ (resp. $\mathbb{R} P^{2}$ ) (see [8, 9, 17]) and one has (see [14] and [16])

$$
\Lambda\left(\mathbb{S}^{2}\right)=\lambda_{1}\left(\mathbb{S}^{2}, g_{\mathbb{S}^{2}}\right) A\left(\mathbb{S}^{2}, g_{\mathbb{S}^{2}}\right)=8 \pi
$$

and

$$
\Lambda\left(\mathbb{R} P^{2}\right)=\lambda_{1}\left(\mathbb{R} P^{2}, g_{\mathbb{R} P^{2}}\right) A\left(\mathbb{R} P^{2}, g_{\mathbb{R} P^{2}}\right)=12 \pi .
$$

Concerning the torus, the flat metrics $g_{s q}$ and $g_{e q}$ associated respectively with the square lattice $\mathbb{Z}^{2}$ and the equilateral lattice $\mathbb{Z}(1,0) \oplus \mathbb{Z}\left(\frac{1}{2}, \frac{\sqrt{3}}{2}\right)$ are, up to dilatations, the only extremal metrics on $\mathbb{T}^{2}$ (see [9]). Nadirashvili [18] has proved the existence of a regular global maximizer of the functional $g \mapsto \lambda_{1}\left(\mathbb{T}^{2}, g\right)$, which then implies that

$$
\Lambda\left(\mathbb{T}^{2}\right)=\lambda_{1}\left(\mathbb{T}^{2}, g_{e q}\right) A\left(\mathbb{T}^{2}, g_{e q}\right)=\frac{8 \pi^{2}}{\sqrt{3}}
$$

However, some steps in Nadirashvili's proof need to be completed as discussed in the recent work of Girouard [13. The metric $g_{s q}$ corresponds to a saddle point of the functional $\lambda_{1}$. 


\section{What about the Klein bottle $\mathbb{K}$ ?}

Nadirashvili [18] observed that an extremal metric on $\mathbb{K}$ cannot be a flat metric. Recently, Jakobson, Nadirashvili and Polterovich [15] proved that a metric of revolution

$$
g_{0}=\frac{9+\left(1+8 \cos ^{2} v\right)^{2}}{1+8 \cos ^{2} v}\left(d u^{2}+\frac{d v^{2}}{1+8 \cos ^{2} v}\right),
$$

$0 \leq u<\frac{\pi}{2}, 0 \leq v<\pi$, is an extremal metric on $\mathbb{K}$ and conjectured that this metric is, up to a dilatation, the unique extremal metric on $\mathbb{K}$.

The main purpose of this paper is to prove this conjecture. Indeed, we will prove the following

Theorem 1.1. The Riemannian metric $g_{0}$ is, up to a dilatation, the unique extremal metric of the functional $\lambda_{1}$ under area preserving deformations of metrics on the Klein bottle $\mathbb{K}$.

Remark 1.1. Nadirashvili 18 has given a sketch of proof of the fact that the supremum $\Lambda(\mathbb{K})$ is necessarily achieved by a regular (real analytic) Riemannian metric. An immediate consequence of such a result and Theorem 1.1 would be

$$
\Lambda(\mathbb{K})=\lambda_{1}\left(\mathbb{K}, g_{0}\right) A\left(\mathbb{K}, g_{0}\right)=12 \pi E(2 \sqrt{2} / 3) \simeq 13.365 \pi,
$$

where $E(2 \sqrt{2} / 3)$ is the complete elliptic integral of the second kind evaluated at $\frac{2 \sqrt{2}}{3}$.

It is worth noticing that the metric $g_{0}$ does not maximize the systole functional $g \mapsto \operatorname{sys}(g)$ (where sys $(g)$ denotes the length of the shortest noncontractible loop) over the set of metrics of fixed area on the Klein bottle (see [3]), while on $\mathbb{R} P^{2}$ and $\mathbb{T}^{2}$, the functionals $\lambda_{1}$ and sys are maximized by the same Riemannian metrics.

The proof of Theorem 1.1 relies on the characterization of extremal metrics in terms of minimal immersions into spheres by the first eigenfunctions. Indeed, a metric $g$ is extremal for $\lambda_{1}$ with respect to area preserving deformations if and only if there exists a family $h_{1}, \cdots, h_{d}$ of first eigenfunctions of $\Delta_{g}$ satisfying $\sum_{i \leq d} d h_{i} \otimes d h_{i}=g$ (see [9, 10]). This last condition actually means that the map $\left(h_{1}, \cdots, h_{d}\right):(M, g) \rightarrow \mathbb{R}^{d}$ is an isometric immersion whose image is a minimal immersed submanifold of a sphere.

As noticed in [15], the surface $\left(\mathbb{K}, g_{0}\right)$ is isometrically and minimally immersed in $\mathbb{S}^{4}$ as the bipolar surface of Lawson's minimal torus $\tau_{3,1}$ defined as the image in $\mathbb{S}^{3}$ of the map

$$
(u, v) \mapsto(\cos v \exp (3 i u), \sin v \exp (i u)) .
$$

In fact, we will prove the following

Theorem 1.2. The minimal surface $\left(\mathbb{K}, g_{0}\right) \hookrightarrow \mathbb{S}^{4}$ is, up to isometries, the only isometrically and minimally immersed Klein bottle into a sphere by its first eigenfunctions.

In 8, Ilias and the first author gave a necessary condition of symmetry for a Riemannian metric to admit isometric immersions into spheres by the first eigenfunctions. On the Klein bottle, this condition amounts to the 
invariance of the metric under the natural $\mathbb{S}^{1}$-action on $\mathbb{K}$. Taking into account this symmetry property and the fact that any metric $g$ is conformally equivalent to a flat one, for which the eigenvalues and the eigenfunctions of the Laplacian are explicitly known, it is of course expected that the existence problem of minimal isometric immersions into spheres by the first eigenfunctions reduces to a second order system of ODEs (see Proposition 2.1). Actually, the substantial part of this paper is devoted to the study of the following second order nonlinear system:

$$
\left\{\begin{array}{l}
\varphi_{1}^{\prime \prime}=\left(1-2 \varphi_{1}^{2}-8 \varphi_{2}^{2}\right) \varphi_{1}, \\
\varphi_{2}^{\prime \prime}=\left(4-2 \varphi_{1}^{2}-8 \varphi_{2}^{2}\right) \varphi_{2},
\end{array}\right.
$$

for which we look for periodic solutions satisfying

$$
\left\{\begin{array}{l}
\varphi_{1} \text { is odd and has exactly two zeros in a period, } \\
\varphi_{2} \text { is even and positive everywhere; }
\end{array}\right.
$$

and the initial conditions

$$
\left\{\begin{array}{l}
\varphi_{1}(0)=\varphi_{2}^{\prime}(0)=0(\text { from parity conditions (2) }), \\
\varphi_{2}(0)=\frac{1}{2} \varphi_{1}^{\prime}(0)=: p \in(0,1] .
\end{array}\right.
$$

Notice that a similar approach is used in 12 where the construction of $\mathbb{S}^{1}$-equivariant minimal tori in $\mathbb{S}^{4}$ and $\mathbb{S}^{1}$-equivariant Willmore tori in $\mathbb{S}^{3}$ is related to a completely integrable Hamiltonian system.

In [15, Jakobson, Nadirashvili and Polterovich proved that the initial value $p=\varphi_{2}(0)=\sqrt{3 / 8}$ corresponds to a periodic solution of (11)-(3) satisfying (2). Based on numerical evidence, they conjectured that this value of $p$ is the only one which corresponds to a periodic solution satisfying (2). As mentioned by them, a computer-assisted proof of this conjecture is extremely difficult, due to the lack of stability of the system.

In Section 3, we provide a complete analytic study of System (1). First, we show that this system admits two independent first integrals (one of them has been already found in [15]). Using a suitable linear change of variables, we show that the system becomes Hamiltonian and, hence, integrable. The general theory of integrable Hamiltonian systems tells us that bounded orbits correspond to periodic or quasi-periodic solutions (see [2]). However, to distinguish periodic solutions from non-periodic ones is not easy in general. Fortunately, our first integrals turn out to be quadratic in the momenta which enables us to apply the classical Bertrand-Darboux-Whittaker Theorem and, therefore, to completely decouple the system by means of a parabolic type change of coordinates $\left(\varphi_{1}, \varphi_{2}\right) \mapsto(u, v)$. We show that, for any $p \neq \sqrt{3} / 2$, the solutions $u$ and $v$ of the decoupled system are periodic. The couple $(u, v)$ is then periodic if and only if the periods of $u$ and $v$ are commensurable. We express the periods of $u$ and $v$ in terms of hyper-elliptic integrals and study their ratio as a function of $p$. The following fact (Proposition 3.1) gives an idea about the complexity of the situation: there exists a countable dense subset $\mathcal{P} \subset(0, \sqrt{3} / 2)$ such that the solution of (11)-(3) corresponding to $p \in(0, \sqrt{3} / 2)$ is periodic if and only if $p \in \mathcal{P}$.

In conclusion, we show that the solution associated with $p=\sqrt{3 / 8}$ is the only periodic one to satisfy Condition (2). 


\section{Preliminaries: Reduction of the problem}

According to [9, 10, a necessary and sufficient condition for a Riemannian metric $g$ on a compact manifold $M$ to be extremal for the functional $\lambda_{1}$ under area-preserving metric deformations is that there exists a family $h_{1}, \cdots, h_{d}$ of first eigenfunctions of $\Delta_{g}$ satisfying

$$
\sum_{i \leq d} d h_{i} \otimes d h_{i}=g
$$

which means that the map $h=\left(h_{1}, \cdots, h_{d}\right)$ is an isometric immersion from $(M, g)$ to $\mathbb{R}^{d}$. Since $h_{1}, \cdots, h_{d}$ are eigenfunctions of $\Delta_{g}$, the image of $h$ is a minimal immersed submanifold of the Euclidean sphere $\mathbb{S}^{d-1}\left(\sqrt{\frac{2}{\lambda_{1}(M, g)}}\right)$ of radius $\sqrt{2 / \lambda_{1}(M, g)}$ (Takahashi's theorem [20]). In particular, we have

$$
\sum_{i \leq d} h_{i}^{2}=\frac{2}{\lambda_{1}(M, g)}
$$

In [8], Ilias and the first author have studied conformal properties of Riemannian manifolds $(M, g)$ admitting such minimal isometric immersions into spheres. It follows from their results that, if $g$ is an extremal metric of $\lambda_{1}$ under area preserving deformations, then

(i) $g$ is, up to a dilatation, the unique extremal metric in its conformal class,

(ii) $g$ maximizes the restriction of $\lambda_{1}$ to the set of metrics conformal to $g$ and having the same volume,

(iii) the isometry group of $(M, g)$ contains the isometry groups of all the metrics $g^{\prime}$ conformal to $g$.

For any positive real number $a$, we denote by $\Gamma_{a}$ the rectangular lattice of $\mathbb{R}^{2}$ generated by the vectors $(2 \pi, 0)$ and $(0, a)$ and by $\tilde{g}_{a}$ the flat Riemannian metric of the torus $\mathbb{T}_{a}^{2} \simeq \mathbb{R}^{2} / \Gamma_{a}$ associated with the rectangular lattice $\Gamma_{a}$. The Klein bottle $\mathbb{K}$ is then diffeomorphic to the quotient of $\mathbb{T}_{a}^{2}$ by the involution $s:(x, y) \mapsto(x+\pi,-y)$. We denote by $g_{a}$ the flat metric induced on $\mathbb{K}$ by such a diffeomorphism. It is well known that any Riemannian metric on $\mathbb{K}$ is conformally equivalent to one of the flat metrics $g_{a}$.

Let $g=f g_{a}$ be a Riemannian metric on $\mathbb{K}$. From the property (iii) above, if $g$ is an extremal metric of $\lambda_{1}$ under area preserving deformations, then $\operatorname{Isom}\left(\mathbb{K}, g_{a}\right) \subset \operatorname{Isom}(\mathbb{K}, g)$, which implies that the function $f$ is invariant under the $\mathbb{S}^{1}$-action $(x, y) \mapsto(x+t, y), t \in[0, \pi]$, on $\mathbb{K}$, and then, $f$ (or its lift to $\mathbb{R}^{2}$ ) does not depend on the variable $x$.

Proposition 2.1. Let a be a positive real number and $f$ a positive periodic function of period $a$. The following assertions are equivalent

(I) The Riemannian metric $g=f(y) g_{a}$ on $\mathbb{K}$ is an extremal metric of the functional $\lambda_{1}$ under area preserving deformations.

(II) There exists a homothetic minimal immersion $h=\left(h_{1}, \cdots, h_{d}\right)$ : $(\mathbb{K}, g) \rightarrow \mathbb{S}^{d-1}$ such that, $\forall i \leq d, h_{i}$ is first eigenfunction of $\Delta_{g}$.

(III) The function $f$ is proportional to $\varphi_{1}^{2}+4 \varphi_{2}^{2}$, where $\varphi_{1}$ and $\varphi_{2}$ are two periodic functions of period a satisfying the following conditions: 
(a) $\left(\varphi_{1}, \varphi_{2}\right)$ is a solution of the equations

$$
\left\{\begin{array}{l}
\varphi_{1}^{\prime \prime}=\left(1-2 \varphi_{1}^{2}-8 \varphi_{2}^{2}\right) \varphi_{1}, \\
\varphi_{2}^{\prime \prime}=\left(4-2 \varphi_{1}^{2}-8 \varphi_{2}^{2}\right) \varphi_{2}
\end{array}\right.
$$

(b) $\varphi_{1}$ is odd, $\varphi_{2}$ is even and $\varphi_{1}^{\prime}(0)=2 \varphi_{2}(0)$;

(c) $\varphi_{1}$ admits two zeros in a period and $\varphi_{2}$ is positive everywhere;

(d) $\varphi_{1}^{2}+\varphi_{2}^{2} \leq 1$ and the equality holds at exactly two points in a period.

From the results [9, 10] mentioned above, it is clear that (I) and (II) are equivalent. Most of the arguments of the proof of "(II) implies (III)" can be found in [18] and [15]. For the sake of completeness, we will recall the main steps. The proof of "(III) implies (II)" relies on the fact that the system (11) admits two independent first integrals.

Proof of Proposition 2.1. The Laplacian $\Delta_{g}$ associated with the Riemannian metric $g=f(y) g_{a}$ on $\mathbb{K}$ can be identified with the operator $-\frac{1}{f(y)}\left(\partial_{x}^{2}+\partial_{y}^{2}\right)$ acting on $\Gamma_{a}$-periodic and $s$-invariant functions on $\mathbb{R}^{2}$. Using separation of variables and Fourier expansions, one can easily show that any eigenfunction of $\Delta_{g}$ is a linear combination of functions of the form $\varphi_{k}(y) \cos k x$ and $\varphi_{k}(y) \sin k x$, where, $\forall k, \varphi_{k}$ is a periodic function with period $a$ satisfying $\varphi_{k}(-y)=(-1)^{k} \varphi_{k}(y)$ and $\varphi_{k}^{\prime \prime}=\left(k^{2}-\lambda f\right) \varphi_{k}$. Since a first eigenfunction always admits exactly two nodal domains, the first eigenspace of $\Delta_{g}$ is spanned by

$$
\left\{\varphi_{0}(y), \varphi_{1}(y) \cos x, \varphi_{1}(y) \sin x, \varphi_{2}(y) \cos 2 x, \varphi_{2}(y) \sin 2 x\right\},
$$

where, unless they are identically zero, $\varphi_{2}$ does not vanish while $\varphi_{0}$ and $\varphi_{1}$ admit exactly two zeros in $[0, a)$. In particular, the multiplicity of $\lambda_{1}(\mathbb{K}, g)$ is at most 5 .

Let us suppose that $g$ is an extremal metric of $\lambda_{1}$ under area preserving deformations and let $h_{1}, \cdots, h_{d}$ be a family of first eigenfunctions satisfying the equations (44) and (5) above. Without loss of generality, we may assume that $\lambda_{1}(\mathbb{K}, g)=2$ and that $h_{1}, \cdots, h_{d}$ are linearly independent, which implies that $d \leq 5$. Since $h=\left(h_{1}, \cdots, h_{d}\right): \mathbb{K} \rightarrow \mathbb{S}^{d-1}$ is an immersion, one has $d \geq 4$. If $d=4$, then using elementary algebraic arguments like in the proof of Proposition 5 of [17, one can see that there exists an isometry $\rho \in O(4)$ such that $\rho \circ h=\left(\varphi_{1}(y) e^{i x}, \varphi_{2}(y) e^{2 i x}\right)$ with $\varphi_{1}^{2}+\varphi_{2}^{2}=1$ (eq. (5) ) and $\varphi^{\prime 2}+\varphi_{2}^{\prime 2}=\varphi_{1}^{2}+4 \varphi_{2}^{2}=f$ (eq. (44) $)$ which is impossible since $\varphi_{1}^{2}+\varphi_{2}^{2}=1$ implies that $\varphi_{1}$ and $\varphi_{2}$ admit a common critical point. Therefore, $d=$ multiplicity of $\lambda_{1}(\mathbb{K}, g)=5$ and there exists $\rho \in O(5)$ such that $\rho \circ h=$ $\left(\varphi_{0}(y), \varphi_{1}(y) e^{i x}, \varphi_{2}(y) e^{2 i x}\right)$, with $\varphi_{0}^{2}+\varphi_{1}^{2}+\varphi_{2}^{2}=1$ and $\varphi_{0}^{\prime 2}+{\varphi^{\prime}}_{1}^{2}+{\varphi^{\prime}}_{2}^{2}=$ $\varphi_{1}^{2}+4 \varphi_{2}^{2}=f$. Since the linear components of $\rho \circ h$ are first eigenfunctions of $(\mathbb{K}, g)$, one should has, $\forall k=0,1,2, \varphi_{k}^{\prime \prime}=\left(k^{2}-\lambda_{1}(\mathbb{K}, g) f\right) \varphi_{k}=\left(k^{2}-2 \varphi_{1}^{2}-\right.$ $\left.8 \varphi_{2}^{2}\right) \varphi_{k}$. Now, it is immediate to check that one of the couples of functions $\left( \pm \varphi_{1}, \pm \varphi_{2}\right)$ satisfies the Conditions (a), .., (d) of the statement. Indeed, the parity condition $\varphi_{k}(-y)=(-1)^{k} \varphi_{k}(y)$ implies that $\varphi_{1}(0)=\varphi_{0}^{\prime}(0)=$ $\varphi_{2}^{\prime}(0)=0$ and, then, $\varphi_{1}^{\prime 2}(0)=4 \varphi_{2}^{2}(0)$. Conditions (c) and (d) follow from the fact that a first eigenfunction has exactly two nodal domains in $\mathbb{K}$. 
Conversely, let $\varphi_{1}$ and $\varphi_{2}$ be two periodic functions of period $a$ satisfying Conditions (a), .., (d) of (III) and consider the Riemannian metric $g=$ $f(y) g_{a}$ on $\mathbb{K}$, with $f=\varphi_{1}^{2}+4 \varphi_{2}^{2}$. We set $\varphi_{0}=\sqrt{1-\varphi_{1}^{2}-\varphi_{2}^{2}}$ and define the $\operatorname{map} h: \mathbb{K} \rightarrow \mathbb{S}^{4}$ by $h=\left(\varphi_{0}(y), \varphi_{1}(y) e^{i x}, \varphi_{2}(y) e^{2 i x}\right)$. It suffices to check that the components of $h$ are first eigenfunctions of $\Delta_{g}$ satisfying (4).

Indeed, in the next section we will see that the second order differential system satisfied by $\varphi_{1}$ and $\varphi_{2}$ (Condition (a)) admits the two following first integrals:

$$
\left\{\begin{array}{l}
\left(\varphi_{1}^{2}+4 \varphi_{2}^{2}\right)^{2}-\varphi_{1}^{2}-16 \varphi_{2}^{2}+{\varphi_{1}^{\prime}}^{2}+4{\varphi_{2}^{\prime}}^{2}=C \\
12 \varphi_{2}^{2}\left(\varphi_{2}^{2}-1\right)+3 \varphi_{1}^{2} \varphi_{2}^{2}+\varphi_{2}^{2}{\varphi_{1}^{\prime}}^{2}-2 \varphi_{1} \varphi_{1}^{\prime} \varphi_{2} \varphi_{2}^{\prime}+\left(3+\varphi_{1}^{2}\right) \varphi_{2}^{\prime 2}=C
\end{array}\right.
$$

with $C=4 \varphi_{2}(0)^{2}\left(4 \varphi_{2}(0)^{2}-3\right)$ (note that Condition (b) implies that $\varphi_{1}(0)=$ $\left.\varphi_{2}^{\prime}(0)=0\right)$. Differentiating $\varphi_{0}^{2}+\varphi_{1}^{2}+\varphi_{2}^{2}=1$ and using the second equation in (6), we get

$$
\begin{aligned}
\varphi_{0}^{2} \varphi_{0}^{\prime 2} & =\varphi_{1}^{2}{\varphi_{1}^{\prime}}^{2}+\varphi_{2}^{2}{\varphi_{2}^{\prime}}^{2}+2 \varphi_{1} \varphi_{1}^{\prime} \varphi_{2} \varphi_{2}^{\prime} \\
& =\varphi_{1}^{2} \varphi_{1}^{\prime 2}+\varphi_{2}^{2} \varphi_{2}^{\prime 2}+12 \varphi_{2}^{2}\left(\varphi_{2}^{2}-1\right)+3 \varphi_{1}^{2} \varphi_{2}^{2}+\varphi_{2}^{2} \varphi_{1}^{\prime 2}+\left(3+\varphi_{1}^{2}\right) \varphi_{2}^{\prime 2}-C \\
& =\left(\varphi_{1}^{2}+\varphi_{2}^{2}\right) \varphi_{1}^{\prime 2}+\left(3+\varphi_{1}^{2}+\varphi_{2}^{2}\right) \varphi_{2}^{\prime 2}+12 \varphi_{2}^{2}\left(\varphi_{2}^{2}-1\right)+3 \varphi_{1}^{2} \varphi_{2}^{2}-C \\
& =\left(1-\varphi_{0}^{2}\right) \varphi_{1}^{\prime 2}+\left(4-\varphi_{0}^{2}\right) \varphi_{2}^{\prime 2}+12 \varphi_{2}^{2}\left(\varphi_{2}^{2}-1\right)+3 \varphi_{1}^{2} \varphi_{2}^{2}-C .
\end{aligned}
$$

Therefore

$$
\begin{aligned}
\varphi_{0}^{2}\left(\varphi_{0}^{\prime 2}+\varphi_{1}^{\prime 2}+\varphi_{2}^{\prime 2}\right) & =\varphi_{1}^{\prime 2}+4{\varphi_{2}^{\prime}}^{2}+12 \varphi_{2}^{2}\left(\varphi_{2}^{2}-1\right)+3 \varphi_{1}^{2} \varphi_{2}^{2}-C \\
& =\left(1-\varphi_{1}^{2}-\varphi_{2}^{2}\right)\left(\varphi_{1}^{2}+4 \varphi_{2}^{2}\right),
\end{aligned}
$$

where the last equality follows from the first equation of (6). Hence,

$$
\left|\partial_{y} h\right|^{2}=\varphi_{0}^{\prime 2}+\varphi_{1}^{\prime 2}+\varphi_{2}^{\prime 2}=\varphi_{1}^{2}+4 \varphi_{2}^{2}=\left|\partial_{x} h\right|^{2}
$$

and, since $\partial_{x} h$ and $\partial_{y} h$ are orthogonal, the map $h$ is isometric, which means that Equation (4) is satisfied.

From Condition (a) one has $\varphi_{1}^{\prime \prime}=(1-2 f) \varphi_{1}$ and $\varphi_{2}^{\prime \prime}=(4-2 f) \varphi_{2}$, which implies that the functions $h_{1}=\varphi_{1}(y) \cos x, h_{2}=\varphi_{1}(y) \sin x, h_{3}=$ $\varphi_{2}(y) \cos 2 x$ and $h_{4}=\varphi_{2}(y) \sin 2 x$ are eigenfunctions of $\Delta_{g}$ associated with the eigenvalue $\lambda=2$. Moreover, differentiating twice the identity $\varphi_{0}^{2}+$ $\varphi_{1}^{2}+\varphi_{2}^{2}=1$ and using Condition (a) and the identity $\varphi_{0}^{\prime 2}+{\varphi_{1}^{\prime}}^{2}+{\varphi_{2}^{\prime}}^{2}=$ $\varphi_{1}^{2}+4 \varphi_{2}^{2}=f$, one obtains after an elementary computation, $\varphi_{0}^{\prime \prime}=-2 f \varphi_{0}$. Hence, all the components of $h$ are eigenfunctions of $\Delta_{g}$ associated with the eigenvalue $\lambda=2$. It remains to prove that 2 is the first positive eigenvalue of $\Delta_{g}$ or, equivalently, for each $k=0,1,2$, the function $\varphi_{k}$ corresponds to the lowest positive eigenvalue of the Sturm-Liouville problem $\varphi^{\prime \prime}=\left(k^{2}-\lambda f\right) \varphi$ subject to the parity condition $\varphi(-y)=(-1)^{k} \varphi(y)$. As explained in the proof of Proposition 3.4.1 of [15], this follows from conditions (c) and (d) giving the number of zeros of $\varphi_{k}$, and the special properties of the zero sets of solutions of Sturm-Liouville equations (oscillation theorems of Haupt and Sturm).

Remark 2.1. Once the initial conditions $\varphi_{1}^{\prime}(0)=2 \varphi_{2}(0)=p$ and $\varphi_{1}(0)=$ $\varphi_{2}^{\prime}(0)=0$ (since $\varphi_{1}$ is odd and $\varphi_{2}$ is even) are fixed, the solution of the system given in assertion (III) of Proposition 2.1 is clearly unique. Hence, as 
we have seen in the proof of this proposition, if a Klein bottle $\left(\mathbb{K}, g=f(y) g_{a}\right)$ admits an isometric full minimal immersion $h:(\mathbb{K}, g) \rightarrow \mathbb{S}^{d-1}$ by the first eigenfunctions, then $d=$ the multiplicity of $\lambda_{1}(\mathbb{K}, g)=5$ and there exists $\rho \in O(5)$ such that

$$
\rho \circ h=\left(\sqrt{1-\varphi_{1}^{2}(y)-\varphi_{2}^{2}(y)}, \varphi_{1}(y) e^{i x}, \varphi_{2}(y) e^{2 i x}\right),
$$

where $\left(\varphi_{1}, \varphi_{2}\right)$ is a unique solution of (III) (with $\varphi_{1}^{\prime}(0)=2 \varphi_{2}(0)=\sqrt{f(0)}$ and $\left.\varphi_{1}(0)=\varphi_{2}^{\prime}(0)=0\right)$. Recall that an immersion $h$ into $\mathbb{S}^{d-1}$ is said to be full if its image does not lie in any hyperplane of $\mathbb{R}^{d}$ (i.e. its components $h_{1}, \ldots, h_{d}$ are linearly independent).

\section{Study of the DyNAMICAL System: PRoOF of RESUlts}

According to Proposition 2.1, one needs to deal with the following system of second order differential equations (Condition (a) of Prop. 2.1)

$$
\left\{\begin{array}{l}
\varphi_{1}^{\prime \prime}=\left(1-2 \varphi_{1}^{2}-8 \varphi_{2}^{2}\right) \varphi_{1} \\
\varphi_{2}^{\prime \prime}=\left(4-2 \varphi_{1}^{2}-8 \varphi_{2}^{2}\right) \varphi_{2}
\end{array}\right.
$$

subject to the initial conditions (Condition (b) of Prop. 2.1)

$$
\left\{\begin{array}{l}
\varphi_{1}(0)=0, \quad \varphi_{2}(0)=p \\
\varphi_{1}^{\prime}(0)=2 p, \varphi_{2}^{\prime}(0)=0
\end{array}\right.
$$

where $p \in(0,1]$ (Condition (d) of Prop. 2.1).

Notice that the system (7)-(8) is invariant under the transform

$$
\left(\varphi_{1}(y), \varphi_{2}(y)\right) \mapsto\left(-\varphi_{1}(-y), \varphi_{2}(-y)\right) .
$$

Consequently, the solution $\left(\varphi_{1}, \varphi_{2}\right)$ of (7)-(8) is such that $\varphi_{1}$ is odd and $\varphi_{2}$ is even.

We are looking for periodic solutions satisfying the following condition (Condition (c) of Prop. 2.1):

$$
\left\{\begin{array}{l}
\varphi_{1} \text { has exactly two zeros in a period, } \\
\varphi_{2} \text { is positive everywhere. }
\end{array}\right.
$$

Our aim is to prove the following

Theorem 3.1. There exists only one periodic solution of (7)-(8) satisfying Condition (9). It corresponds to the initial value $\varphi_{2}(0)=p=\sqrt{3 / 8}$.

In fact, this theorem follows from the qualitative behavior of solutions, in terms of $p$, given in the following

Proposition 3.1. Let $\left(\varphi_{1}, \varphi_{2}\right)$ be the solution of (7)-(8).

(1) For all $p \in(0,1], p \neq \sqrt{3} / 2,\left(\varphi_{1}, \varphi_{2}\right)$ is periodic or quasi-periodic.

(2) For $p=\frac{\sqrt{3}}{2},\left(\varphi_{1}, \varphi_{2}\right)$ tends to the origin as $y \rightarrow \infty$ (hence, it is neither periodic nor quasi-periodic).

(3) For all $p \in(\sqrt{3} / 2,1], \varphi_{2}$ vanishes at least once in each period (of $\left.\varphi_{2}\right)$. Hence, Condition (9) is not satisfied.

(4) There exists a countable dense subset $\mathcal{P} \subset(0, \sqrt{3} / 2)$, with $\sqrt{3 / 8} \in$ $\mathcal{P}$, such that the solution $\left(\varphi_{1}, \varphi_{2}\right)$ corresponding to $p \in(0, \sqrt{3} / 2)$ is periodic if and only if $p \in \mathcal{P}$. 
(5) For $p=\sqrt{3 / 8},\left(\varphi_{1}, \varphi_{2}\right)$ satisfies (9) and, for any $p \in \mathcal{P}, p \neq \sqrt{3 / 8}$, $\varphi_{1}$ admits at least 6 zeros in a period.

Notice that the assertions (2) and (3) of this proposition were also proved in [15] by other methods.

The first fundamental step in the study of the system above is the existence of the following two independent first integrals.

3.1. First integrals. The functions

$$
\left\{\begin{array}{c}
H_{1}\left(\varphi_{1}, \varphi_{2}, \varphi_{1}^{\prime}, \varphi_{2}^{\prime}\right):=\left(\varphi_{1}^{2}+4 \varphi_{2}^{2}\right)^{2}-\varphi_{1}^{2}-16 \varphi_{2}^{2}+\left(\varphi_{1}^{\prime}\right)^{2}+4\left(\varphi_{2}^{\prime}\right)^{2} \\
H_{2}\left(\varphi_{1}, \varphi_{2}, \varphi_{1}^{\prime}, \varphi_{2}^{\prime}\right):=12 \varphi_{2}^{2}\left(\varphi_{2}^{2}-1\right)+3 \varphi_{1}^{2} \varphi_{2}^{2}+\varphi_{2}^{2}\left(\varphi_{1}^{\prime}\right)^{2} \\
-2 \varphi_{1} \varphi_{1}^{\prime} \varphi_{2} \varphi_{2}^{\prime}+\left(3+\varphi_{1}^{2}\right)\left(\varphi_{2}^{\prime}\right)^{2}
\end{array}\right.
$$

are two independent first integrals of (7), i.e. they satisfy the equation

$$
\varphi_{1}^{\prime} \frac{\partial H_{i}}{\partial \varphi_{1}}+\varphi_{2}^{\prime} \frac{\partial H_{i}}{\partial \varphi_{2}}+\varphi_{1}^{\prime \prime} \frac{\partial H_{i}}{\partial \varphi_{1}^{\prime}}+\varphi_{2}^{\prime \prime} \frac{\partial H_{i}}{\partial \varphi_{2}^{\prime}} \equiv 0 .
$$

The first one, $H_{1}$, has been obtained by Jakobson et al. [15]. The orbit of a solution of (7) is then contained in an algebraic variety defined by

$$
\left\{\begin{array}{l}
H_{1}\left(\varphi_{1}, \varphi_{2}, \varphi_{1}^{\prime}, \varphi_{2}^{\prime}\right)=K_{1}, \\
H_{2}\left(\varphi_{1}, \varphi_{2}, \varphi_{1}^{\prime}, \varphi_{2}^{\prime}\right)=K_{2},
\end{array}\right.
$$

where $K_{1}$ and $K_{2}$ are two constants. Taking into account the initial conditions (8), one has $K_{1}=K_{2}=-4 p^{2}\left(3-4 p^{2}\right)$. In other words, the solution of (77)-(8) is also solution of

$$
\left\{\begin{array}{c}
\left(\varphi_{1}^{2}+4 \varphi_{2}^{2}\right)^{2}-\varphi_{1}^{2}-16 \varphi_{2}^{2}+\left(\varphi_{1}^{\prime}\right)^{2}+4\left(\varphi_{2}^{\prime}\right)^{2}+4 p^{2}\left(3-4 p^{2}\right)=0 \\
12 \varphi_{2}^{2}\left(\varphi_{2}^{2}-1\right)+3 \varphi_{1}^{2} \varphi_{2}^{2}+\varphi_{2}^{2}\left(\varphi_{1}^{\prime}\right)^{2}-2 \varphi_{1} \varphi_{1}^{\prime} \varphi_{2} \varphi_{2}^{\prime} \\
+\left(3+\varphi_{1}^{2}\right)\left(\varphi_{2}^{\prime}\right)^{2}+4 p^{2}\left(3-4 p^{2}\right)=0
\end{array}\right.
$$

with the initial conditions

$$
\left\{\begin{array}{l}
\varphi_{1}(0)=0, \\
\varphi_{2}(0)=p .
\end{array}\right.
$$

Notice that the parameter $p$ appears in both the equations (12) and the initial conditions (13). The system (12) gives rise to a "multi-valued" 2dimensional dynamical system in the following way.

3.2. 2-dimensional dynamical systems. From (12) one can extract explicit expressions of $\varphi_{1}^{\prime}$ and $\varphi_{2}^{\prime}$ in terms of $\varphi_{1}$ and $\varphi_{2}$. For instance, eliminating $\varphi_{1}^{\prime}$, one obtains the following fourth degree equation in $\varphi_{2}^{\prime}$

$$
d_{4}\left(\varphi_{1}, \varphi_{2}\right)\left(\varphi_{2}^{\prime}\right)^{4}-2 d_{2}\left(\varphi_{1}, \varphi_{2}\right)\left(\varphi_{2}^{\prime}\right)^{2}+d_{0}\left(\varphi_{1}, \varphi_{2}\right)=0,
$$

where $d_{0}, d_{2}$ and $d_{4}$ are polynomials in $\varphi_{1}, \varphi_{2}$ and $p$. The discriminant of (14) is given by

$$
\Delta:=-64 \varphi_{1}^{2} \varphi_{2}^{2} w_{1} w_{2} w_{3},
$$


with

$$
\begin{aligned}
& w_{1}\left(\varphi_{1}, \varphi_{2}\right)=\varphi_{1}^{2}+\varphi_{2}^{2}-1, \\
& w_{2}\left(\varphi_{1}, \varphi_{2}\right)=p^{2} \varphi_{1}^{2}-\left(3-4 p^{2}\right) \varphi_{2}^{2}+p^{2}\left(3-4 p^{2}\right), \\
& w_{3}\left(\varphi_{1}, \varphi_{2}\right)=-\left(3-4 p^{2}\right) \varphi_{1}^{2}+16 p^{2} \varphi_{2}^{2}-4 p^{2}\left(3-4 p^{2}\right) .
\end{aligned}
$$

It is quite easy to show that, for any $p$, each one of the curves $\left(w_{i}=0\right)$ contains the orbit of a particular solution of (12). Moreover, the unit circle $\left(w_{1}=0\right)$ represents the orbit of the solution of (12) satisfying the initial conditions (13) with $p=1$. For $p=\sqrt{3 / 8}$, we have $w_{3} \equiv-4 w_{2}$ and the curve $\left(w_{2}=0\right)$ contains the orbit of the solution of (12)-(13).

These particular algebraic orbits suggest us searching solutions $\left(\varphi_{1}, \varphi_{2}\right)$ defined by algebraic relations of the form $w_{4}\left(\varphi_{1}, \varphi_{2}\right)=F\left(\varphi_{1}^{2}, \varphi_{2}^{2}\right)=0$, where $F$ is a polynomial of degree $\leq 4$. Apart the three quadrics above, the only additional solution of this type we found is

$$
w_{4}=\left(\varphi_{1}^{2}+4 \varphi_{2}^{2}\right)^{2}-12 \varphi_{2}^{2}=0 .
$$

Like $\left(w_{1}=0\right)$, the curve $\left(w_{4}=0\right)$ is independent of $p$ and represents the orbit of a particular solution of (12) for arbitrary values of $p$. Since

$$
w_{4}\left(\varphi_{1}, \varphi_{2}\right)=\left(\varphi_{1}^{2}+4 \varphi_{2}^{2}-2 \sqrt{3} \varphi_{2}\right)\left(\varphi_{1}^{2}+4 \varphi_{2}^{2}+2 \sqrt{3} \varphi_{2}\right),
$$

the set $\left(w_{4}=0\right)$ is the union of two ellipses passing through the origin, each one being symmetric to the other with respect to the $\varphi_{1}$-axis. The upper ellipse

$$
\varphi_{1}^{2}+4 \varphi_{2}^{2}-2 \sqrt{3} \varphi_{2}=0
$$

corresponds to the orbit of the solution of (12)-(13) associated with $p=\frac{\sqrt{3}}{2}$.

3.3. Proof of Proposition 3.1)(2): case $p=\frac{\sqrt{3}}{2}$. In this case, the orbit of the solution of (12)-(13) is given by (15). The only critical point of (12) lying on this ellipse is the origin, which is also a critical point of the system (77). Therefore, $\left(\varphi_{1}(y), \varphi_{2}(y)\right)$ tends to the origin as $y$ goes to infinity (see also [15]).

From now on, we will assume that $p \neq \frac{\sqrt{3}}{2}$.

3.4. A bounded region for the orbit. The orbit of the solution of (12)(13) must lie in the region of the $\left(\varphi_{1}, \varphi_{2}\right)$-plane where the discriminant $\Delta$ of (14) is nonnegative. This region, $(\Delta \geq 0)$, is a bounded domain delimited by the unit circle $\left(w_{1}=0\right)$ and the quadrics $\left(w_{2}=0\right)$ and $\left(w_{3}=0\right)$. Its shape depends on the values of $p$.

- For $p \in(0, \sqrt{3} / 2),\left(w_{2}=0\right)$ and $\left(w_{3}=0\right)$ are hyperbolas.

- The case $p=\sqrt{3 / 8}$ is a special one since then, $w_{3} \equiv-4 w_{2}$, and the region $(\Delta \geq 0)$ shrinks to the arc of the hyperbola $\left(w_{2}=0\right)$ lying inside the unit disk.

- For $p \in(\sqrt{3} / 2,1], w_{3}$ is positive and $\left(w_{2}=0\right)$ is an ellipse.

From (14) and (12) one can express $\varphi_{1}^{\prime}$ and $\varphi_{2}^{\prime}$ in terms of $\varphi_{1}, \varphi_{2}$ and $p$. Thus, we obtain a multi-valued 2-dimensional dynamical system parameterized by $p$ with the initial conditions $\varphi_{1}(0)=0$ and $\varphi_{2}(0)=p$. However, the dynamics of such a multi-valued system is very complex to study. 
Fortunately, as we will see in the next subsections, the system (7) can be transformed, by means of a suitable change of variables, into a Hamiltonian system, completely integrable by quadratures.

3.5. Hamiltonian dynamical system. Let us introduce the new variables $q_{1}$ and $q_{2}$ defined by

$$
q_{1}:=\frac{1}{\sqrt{2}} \varphi_{1} \quad, \quad q_{2}:=\sqrt{2} \varphi_{2} .
$$

The system (7) becomes

$$
\left\{\begin{array}{l}
q_{1}^{\prime \prime}=\left[1-4\left(q_{1}^{2}+q_{2}^{2}\right)\right] q_{1}=-\frac{\partial V}{\partial q_{1}}, \\
q_{2}^{\prime \prime}=4\left[1-q_{1}^{2}-q_{2}^{2}\right] q_{2}=-\frac{\partial V}{\partial q_{2}},
\end{array}\right.
$$

with

$$
V\left(q_{1}, q_{2}\right):=\left(q_{1}^{2}+q_{2}^{2}\right)^{2}-\frac{1}{2} q_{1}^{2}-2 q_{2}^{2} .
$$

Therefore, one has a Hamiltonian system with two degrees of freedom. The Hamiltonian $H$ is given by

$$
H\left(q_{1}, q_{2}, q_{1}^{\prime}, q_{2}^{\prime}\right):=\frac{1}{2}\left[\left(q_{1}^{\prime}\right)^{2}+\left(q_{2}^{\prime}\right)^{2}\right]+V\left(q_{1}, q_{2}\right) .
$$

This Hamiltonian is a first integral of (17) (notice that $H=\frac{1}{4} H_{1}$ ). A second independent first integral of (17) can be obtained from $\mathrm{H}_{2}$. Consequently, the Hamiltonian system (17) is integrable and all its bounded orbits in phase space $\left(q_{1}, q_{2}, q_{1}^{\prime}, q_{2}^{\prime}\right)$ are contained in a 2-dimensional topological torus (see [2]), which means that the corresponding solutions are periodic or quasiperiodic, provided that there is no critical point in the closure of the orbit. However, it is in general difficult to decide whether such a solution is periodic or not. The corresponding topological torus obtained from (12) is given by:

$$
\left\{\begin{array}{c}
\frac{1}{2}\left[\left(q_{1}^{\prime}\right)^{2}+\left(q_{2}^{\prime}\right)^{2}\right]+\left(q_{1}^{2}+q_{2}^{2}\right)^{2}-\frac{1}{2} q_{1}^{2}-2 q_{2}^{2}+p^{2}\left(3-4 p^{2}\right)=0 \\
3 q_{2}^{2}\left(q_{2}^{2}-2\right)+3 q_{1}^{2} q_{2}^{2}+\left(q_{1}^{\prime}\right)^{2} q_{2}^{2}-2 q_{1} q_{1}^{\prime} q_{2} q_{2}^{\prime} \\
+\frac{1}{2}\left(3+2 q_{1}^{2}\right)\left(q_{2}^{\prime}\right)^{2}+4 p^{2}\left(3-4 p^{2}\right)=0 .
\end{array}\right.
$$

It is important to notice that the second first integral is also quadratic in the $q_{1}^{\prime}$ and $q_{2}^{\prime}$ variables. Indeed, this enables us to apply the BertrandDarboux-Whittaker theorem: Given a Hamiltonian system defined by

$$
H=\frac{1}{2}\left[\left(q_{1}\right)^{\prime 2}+\left(q_{2}^{\prime}\right)^{2}\right]+V\left(q_{1}, q_{2}\right),
$$

the system admits an additional independent first integral, quadratic in $q_{1}^{\prime}$ and $q_{2}^{\prime}$, if and only if the system is separable in cartesian, polar, parabolic, or elliptic-hyperbolic coordinates. (see [1, 21] for details).

In our case, an adequate change of variables is a parabolic one, given by

$$
\left\{\begin{array}{l}
q_{1}^{2}=-\frac{2}{3} u v \\
q_{2}^{2}=\frac{1}{6}(3+2 u)(3+2 v) .
\end{array}\right.
$$


Indeed, from (18), one obtains after an elementary computation

$$
\left\{\begin{array}{l}
\left(u^{\prime}\right)^{2}=\frac{P(u)}{(u-v)^{2}} \\
\left(v^{\prime}\right)^{2}=\frac{P(v)}{(u-v)^{2}}
\end{array}\right.
$$

where $P(s):=s(1-2 s)(3+2 s)\left(2 p^{2}+s\right)\left(3-4 p^{2}+2 s\right)$. Observe that (20) is not completely decoupled yet; this can be done by means of a suitable change of the independent variable (see Subsection 3.6). Each one of the quadrics $\left(w_{1}=0\right),\left(w_{2}=0\right)$ and $\left(w_{3}=0\right)$ is transformed into two parallel lines. Indeed, we have $w_{1}(u, v)=-\frac{1}{4}(1-2 u)(1-2 v), w_{2}(u, v)=-\left(\frac{3}{2}-\right.$ $\left.2 p^{2}+u\right)\left(\frac{3}{2}-2 p^{2}+v\right)$ and $w_{3}(u, v)=4\left(2 p^{2}+u\right)\left(2 p^{2}+v\right)$. Also, we have $\Delta=\frac{16}{9} P(u) P(v)$. Thus, the region $(\Delta \geq 0)$ is transformed into the region $(P(u) P(v) \geq 0)$.

Observe that the system (20) is symmetric in $u$ and $v$. As the change of variables (19) is also symmetric in $u$ and $v$, and since $u v$ must be non positive, one can assume, without loss of generality, that $u \geq 0$ and, hence, $-\frac{3}{2} \leq v \leq 0$. Now, the condition $(\Delta \geq 0)$ implies that $(u, v) \in I_{1} \times I_{2}$, where

$$
I_{1}:=\left[\alpha_{0}, 1 / 2\right]:= \begin{cases}{\left[0, \frac{1}{2}\right]} & \text { if } p^{2}<\frac{3}{4}, \\ {\left[2 p^{2}-\frac{3}{2}, \frac{1}{2}\right]} & \text { if } \frac{3}{4}<p^{2} \leq 1,\end{cases}
$$

and

$$
I_{2}:=\left[a_{0}, a_{1}\right]:= \begin{cases}{\left[2 p^{2}-\frac{3}{2},-2 p^{2}\right]} & \text { if } p^{2} \leq \frac{3}{8}, \\ {\left[-2 p^{2}, 2 p^{2}-\frac{3}{2}\right]} & \text { if } \frac{3}{8} \leq p^{2}<\frac{3}{4}, \\ {\left[-\frac{3}{2}, 0\right]} & \text { for } \frac{3}{4}<p^{2} \leq 1 .\end{cases}
$$

The initial conditions (13) become

$$
(u(0), v(0))= \begin{cases}\left(0,2 p^{2}-\frac{3}{2}\right) & \text { if } p^{2}<\frac{3}{4}, \\ \left(2 p^{2}-\frac{3}{2}, 0\right) & \text { if } \frac{3}{4}<p^{2} \leq 1 .\end{cases}
$$

In all cases, $u(0)$ and $v(0)$ are zeros of $P$. Hence

$$
\left(u^{\prime}(0), v^{\prime}(0)\right)=(0,0) .
$$

The behavior of $(u, v)$ near $y=0$ is then determined by the acceleration vector

$$
\left(u^{\prime \prime}(0), v^{\prime \prime}(0)\right)= \begin{cases}\left(\frac{12 p^{2}}{3-4 p^{2}}, \frac{16 p^{2}\left(1-p^{2}\right)\left(3-8 p^{2}\right)}{\left(3-4 p^{2}\right)}\right) & \text { if } p^{2}<\frac{3}{4}, \\ \left(\frac{16 p^{2}\left(1-p^{2}\right)\left(3-8 p^{2}\right)}{\left(3-4 p^{2}\right)}, \frac{12 p^{2}}{3-4 p^{2}}\right) & \text { if } \frac{3}{4}<p^{2} \leq 1 .\end{cases}
$$

Notice that for $p=\sqrt{\frac{3}{8}}, v$ is constant, namely $v(y)=-\frac{3}{4}$ for all $y$, while for $p=1, u$ is constant with $u(y)=\frac{1}{2}$ for all $y$. 
3.6. Proof of Proposition 3.1(1): Decoupling the system. In order to completely decouple the previous system we introduce a change of the independent variable $y \mapsto \tau$ defined by:

$$
\frac{d \tau}{d y}=\frac{1}{u-v}
$$

Notice that this change of variable is one-to-one since $u-v \neq 0$ (indeed, $\left.I_{1} \cap I_{2}=\emptyset\right)$. In this new variable, the system splits into two independent equations:

$$
\begin{aligned}
& (\dot{u})^{2}=P(u), \\
& (\dot{v})^{2}=P(v),
\end{aligned}
$$

where $\dot{u}:=d u / d \tau$ and $\dot{v}:=d v / d \tau$. The solution $\tau \mapsto u(\tau)$ of (23) is also a solution of the second order ODE

$$
\ddot{u}=\frac{1}{2} P^{\prime}(u),
$$

with the initial conditions $u(0)=\alpha_{0}$ (see (21) for the definition of $\left.\alpha_{0}\right)$ and $\dot{u}(0)=0$, where $P^{\prime}:=d P / d u$. This solution lies on the curve

$$
(\dot{u})^{2}-P(u)=0
$$

in the $(u, \dot{u})$-phase plane of (25). Since $\alpha_{0}$ and $\frac{1}{2}$ are two consecutive zeros of $P$, the equation (26) in the region $\alpha_{0} \leq u \leq \frac{1}{2}$ represents a closed curve. On the other hand, it is easy to check that $P$ and $P^{\prime}$ admit no common zero in the interval $\left[\alpha_{0}, \frac{1}{2}\right]$. Hence, there exists no critical point for (25) on the orbit defined by (26) inside the region $\alpha_{0} \leq u \leq \frac{1}{2}$. Consequently, this closed orbit corresponds to a periodic solution of (25) and, therefore, $\tau \mapsto u(\tau)$ oscillates between $\alpha_{0}$ and $\frac{1}{2}$.

A similar analysis for $\tau \mapsto v(\tau)$ implies that it is a periodic solution of

$$
\ddot{v}=\frac{1}{2} P^{\prime}(v)
$$

with the initial conditions $v(0)=a_{0}$ (see (22) for the definition of $a_{0}$ ) and $\dot{v}(0)=0$. Consequently, $\tau \mapsto v(\tau)$ oscillates between $a_{0}$ and $a_{1}$. This proves Assertion (1) of Proposition 3.1(1).

3.7. Proof of Proposition [3.1)(3): case $\mathbf{p}>\frac{\sqrt{\mathbf{3}}}{\mathbf{2}}$. We have just seen that $v(\mathbb{R})=\left[a_{0}, a_{1}\right]$, with $a_{0}=-\frac{3}{2}$ for $p \in\left(\frac{\sqrt{3}}{2}, 1\right]$ (see (22) $)$. This implies that $q_{2}$, and then $\varphi_{2}$, vanishes at least once in a period (see (19)).

3.8. About the periods of $u$ and $v$ : case $\mathbf{p}<\frac{\sqrt{3}}{2}$. Let us denote $\mathcal{T}_{u}(p)$ the period of $u$. The function $\tau \mapsto u(\tau)$ oscillates between $\alpha_{0}=0$ and $\frac{1}{2}$ with velocity $(\dot{u})^{2}=P(u) \neq 0$ if $u \in\left(0, \frac{1}{2}\right)$. Hence, $u(\tau)$ increases from 0 to $\frac{1}{2}$ when $\tau$ goes from 0 to $\frac{\mathcal{T}_{u}(p)}{2}$. It follows that

$$
\mathcal{T}_{u}(p)=2 \int_{0}^{\frac{1}{2}} \frac{d s}{\sqrt{P(s)}} .
$$


Similarly, for $p \neq \sqrt{3 / 8}$, the period $\mathcal{T}_{v}(p)$ of $\tau \mapsto v(\tau)$ is given by

$$
\mathcal{T}_{v}(p)=2 \int_{a_{0}}^{a_{1}} \frac{d s}{\sqrt{P(s)}} .
$$

Setting, for $p \neq \sqrt{3 / 8}, s=\left(3-8 p^{2}\right) r-\frac{3}{2}+2 p^{2}$, one can write

$$
\mathcal{T}_{v}(p)=2 \int_{0}^{\frac{1}{2}} \frac{d r}{\sqrt{Q(r)}}
$$

where

$Q(r):=2 r(1-2 r)\left[2 p^{2}+\left(3-8 p^{2}\right) r\right]\left[2-2 p^{2}-\left(3-8 p^{2}\right) r\right]\left[3-4 p^{2}-2\left(3-8 p^{2}\right) r\right]$.

Hence, the functions $\mathcal{T}_{u}(p)$ and $\mathcal{T}_{v}(p)$ are explicitly given by complete hyperelliptic integrals. Although the function $\mathcal{T}_{v}$ is not defined at $p=\sqrt{3 / 8}$, its limit exists. Indeed, setting $t=\frac{3}{4}+r$ and $\alpha:=\frac{3}{4}-2 p^{2}$, we get

$$
\mathcal{T}_{v}(p)=2 \int_{-\alpha}^{\alpha} \frac{d t}{\sqrt{\left(\frac{3}{2}-2 t\right)\left(\frac{5}{2}-2 t\right)\left(\frac{3}{2}+2 t\right)} \sqrt{\alpha^{2}-t^{2}}} .
$$

As $\alpha \rightarrow 0$, we have

$$
\mathcal{T}_{v}(p) \sim 2 \int_{-\alpha}^{\alpha} \frac{4 d t}{3 \sqrt{10} \sqrt{\alpha^{2}-t^{2}}}
$$

Thus

$$
\lim _{p \rightarrow \sqrt{\frac{3}{8}}} \mathcal{T}_{v}(p)=\frac{8 \pi}{3 \sqrt{10}} .
$$

On the other hand, we have

$$
\mathcal{T}_{u}(\sqrt{3 / 8})=\frac{4}{5} \Pi(2 / 5,1 / 4),
$$

where $\Pi$ is the complete elliptic integral of the third kind given by

$$
\Pi(n, m):=\int_{0}^{\frac{\pi}{2}} \frac{d \theta}{\left(1-n \sin ^{2} \theta\right) \sqrt{1-m \sin ^{2} \theta}} .
$$

Since for $p=\sqrt{3 / 8}, v$ is constant, the couple $(u, v)$ is periodic of period $\mathcal{T}_{u}(\sqrt{3 / 8})$.

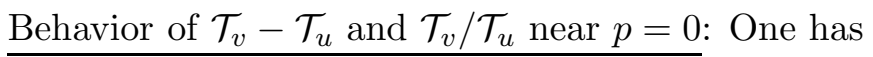

$$
\mathcal{T}_{v}(p)-\mathcal{T}_{u}(p)=2 \int_{0}^{\frac{1}{2}}\left[\frac{1}{\sqrt{Q(s)}}-\frac{1}{\sqrt{P(s)}}\right] d s .
$$

The integral of $\frac{1}{\sqrt{P(s)}}$ is singular only at $p=0$. A direct computation gives

$$
\int_{0}^{\frac{1}{2}} \frac{d s}{\sqrt{P(s)}} \sim \int_{0}^{\frac{1}{2}} \frac{d s}{3 \sqrt{s^{2}+2 p^{2} s}} \sim-\frac{2}{3} \ln (p) .
$$

Similarly, we get

$$
\int_{0}^{\frac{1}{2}} \frac{d s}{\sqrt{Q(s)}} \sim-\ln p
$$


In other words $\mathcal{T}_{v}(p)-\mathcal{T}_{u}(p) \rightarrow+\infty$ as $p \rightarrow 0$ while the ratio $\mathcal{T}_{v}(p) / \mathcal{T}_{u}(p)$ goes to $\frac{3}{2}$ (see the figure below).

Behavior of $\mathcal{T}_{v}-\mathcal{T}_{u}$ and $\mathcal{T}_{v} / \mathcal{T}_{u}$ near $p=\sqrt{3} / 2$ : One has, as $p \rightarrow \sqrt{3} / 2, \mathcal{T}_{u}(p) \sim$ $-\frac{2}{3} \ln (\sqrt{3} / 2-p)$ and $\mathcal{T}_{v}(p) \sim-\ln (\sqrt{3} / 2-p)$. Hence, $\mathcal{T}_{v}-\mathcal{T}_{u} \rightarrow+\infty$ as $p \rightarrow \sqrt{3} / 2$ and $\mathcal{T}_{v}(p) / \mathcal{T}_{u}(p)$ goes again to $\frac{3}{2}$.

Thus, $\mathcal{T}_{v}(p)>\mathcal{T}_{u}(p)$ near $p=0$ and $p=\sqrt{3} / 2$. Actually, one has $\mathcal{T}_{v}(p)>$ $\mathcal{T}_{u}(p)$ for all $p \in(0, \sqrt{3} / 2)$ as shown by the graphic representation of $\mathcal{T}_{v}$ and $\mathcal{T}_{u}$ given below.

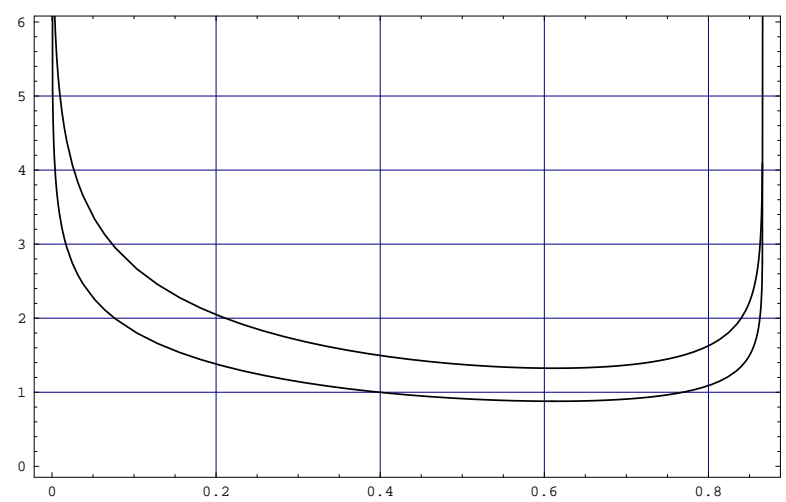

The functions $p \mapsto \mathcal{T}_{v}(p)$ (the upper one) and $p \mapsto \mathcal{T}_{u}(p)$.

3.9. Proof of Proposition 3.1(4). The couple $(u, v)$ is periodic if and only if the ratio $R(p):=\frac{\mathcal{T}_{v}(p)}{\mathcal{T}_{u}(p)}$ is a rational number. From the previous subsection, $R$ is a nonconstant continuous function on $(0, \sqrt{3} / 2)$ with $\lim _{p \rightarrow 0} R(p)=\lim _{p \rightarrow \sqrt{3} / 2} R(p)=3 / 2$. The range of $R$ is a closed interval $\left[r_{1}, r_{2}\right] \subset[1.480473,1.507784]$ (see the figure below).

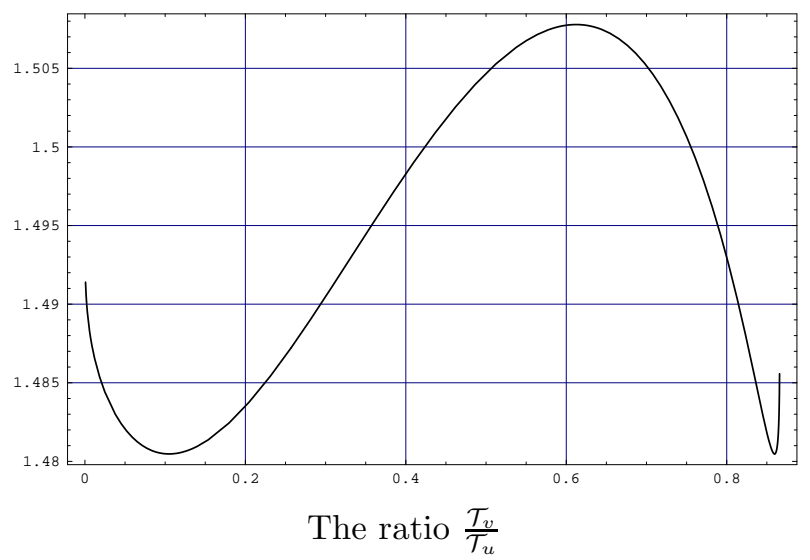

To end the proof of Assertion (4), we only need to define $\mathcal{P}$ to be the set of $p \in(0, \sqrt{3} / 2)$ such that $R(p)$ is a rational number.

3.10. Proof of Proposition 3.1(5). In the case $p=\sqrt{3 / 8}$ we have, $\forall y \in$ $\mathbb{R}, v(y)=-\frac{3}{4}$ and, then, $\varphi_{1}^{2}=u$ and $\varphi_{2}^{2}=\frac{1}{8}(3+2 u)$. The couple of periodic functions $\left(\varphi_{1}, \varphi_{2}\right)=\left(\sqrt{u}, \sqrt{\frac{1}{8}(3+2 u)}\right)$ on $\left[0, \mathcal{T}_{u}(\sqrt{3 / 8})\right]$, such that $\varphi_{1}$ is odd and $\varphi_{2}$ is even, solves the original system and satisfies Condition (9). 
Let $p \in \mathcal{P}, p \neq \sqrt{3 / 8}$, and let $\frac{q}{m} \in \mathbb{Q}$ be an irreducible fraction, with $q$, $m \in \mathbb{N}$, such that $R(p)=\frac{\mathcal{T}_{v}(p)}{\mathcal{T}_{u}(p)}=\frac{q}{m}$. The period of the couple $(u, v)$ is given by

$$
\mathcal{T}(p)=q \mathcal{T}_{u}(p)=m \mathcal{T}_{v}(p) .
$$

The number of zeros of $u$ in a period, for instance $[0, \mathcal{T}(p))$, of $(u, v)$ is equal to $q$ times the number of zeros of $u$ in $\left[0, \mathcal{T}_{u}(p)\right)$. As we saw above, $\forall p \in(0, \sqrt{3} / 2)$, one has $1<R(p)<2$. Hence, $m \geq 2$ and $q>m$, which implies $q \geq 3$. Since $u(0)=0$, the number of zeros of $u$ in a period of $(u, v)$ is at least 3. Since $\varphi_{1}$ is odd, the period of $\left(\varphi_{1}, \varphi_{2}\right)$ is twice the period of $(u, v)$ (see (19). From $\varphi_{1}=2 \sqrt{-\frac{1}{3} u v}$ on $\left[0, \mathcal{T}(p)\right.$ ), one deduces that $\varphi_{1}$ admits at least 6 zeros in a period of $\left(\varphi_{1}, \varphi_{2}\right)$. Notice that the case $p=\sqrt{3 / 8}$ is special since, for this value of $p, v$ is constant and the couple $(u, v)$ is periodic whose period is equal to that of $u$.

3.11. On the shape of solutions. Although it is not necessary for the proof of our results, one can obtain as a by product of our study, some properties concerning the shape of solutions. First, notice that, for $p \in\left(0, \frac{\sqrt{3}}{2}\right)$, the 2-dimensional dynamical system admits four critical points in the region $(\Delta \geq 0) \cap\left(\varphi_{2} \geq 0\right): A=\left(2 p / \sqrt{3}, \sqrt{1-4 p^{2} / 3}\right), B=\left(\sqrt{1-4 p^{2} / 3}, 2 p / \sqrt{3}\right)$ and their symmetric with respect to the $\varphi_{2}$-axis that we denote $A^{\prime}$ and $B^{\prime}$. Notice that these critical points are on the boundary of the region $(\Delta \geq 0)$. Non-periodic solutions. They correspond to the case where $\mathcal{T}_{v}(p) / \mathcal{T}_{u}(p)$ is

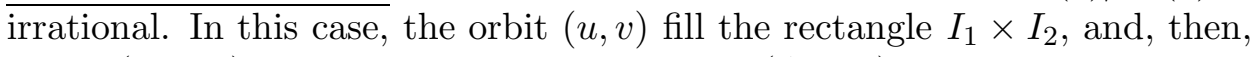
in the $\left(\varphi_{1}, \varphi_{2}\right)$-plane, the orbit fill the region $(\Delta \geq 0)$.

Periodic solutions. For $p=\sqrt{3 / 8}$, the solution $\left(\varphi_{1}, \varphi_{2}\right)$ lies on the hyperbola of equation $\varphi_{1}^{2}-4 \varphi_{2}^{2}+3 / 2=0$, oscillating between the points $A=\left(-\frac{1}{\sqrt{2}}, \frac{1}{\sqrt{2}}\right)$ and $A^{\prime}=\left(\frac{1}{\sqrt{2}}, \frac{1}{\sqrt{2}}\right)$. For $p \neq \sqrt{3 / 8}$, let $q / m=\mathcal{T}_{v}(p) / \mathcal{T}_{u}(p)$ be an irreducible fraction, with $q, m \in \mathbb{N}$, and set $\mathcal{T}:=q \mathcal{T}_{u}(p)=m \mathcal{T}_{v}(p)$. Geometrically, this means that $u$ makes $q$ round trips in a period while $v$ makes $m$ round trips. We distinguish three cases:

- If $q$ and $m$ are both odd, then $u(\mathcal{T} / 2)=\frac{1}{2}$ and $v(\mathcal{T} / 2)=a_{1}$. This corresponds to the point $A$ for $p^{2}<\frac{3}{8}$ and to the point $B$ for $\frac{3}{8}<$ $p^{2}<\frac{3}{4}$. The orbit is not closed and $\left(\varphi_{1}, \varphi_{2}\right)$ oscillates between $A$ and $A^{\prime}$ or $B$ and $B^{\prime}$.

- If $q$ is odd and $m$ is even, then $u(\mathcal{T} / 2)=\frac{1}{2}$ and $v(\mathcal{T} / 2)=a_{0}$. This corresponds to the point $B$ for $p^{2}<\frac{3}{8}$ and to the point $A$ for $\frac{3}{8}<p^{2}<\frac{3}{4}$. Again, $\left(\varphi_{1}, \varphi_{2}\right)$ oscillates between $A$ and $A^{\prime}$ or $B$ and $B^{\prime}$.

- If $q$ is even and $m$ is odd, then $u(\mathcal{T} / 2)=0$ and $v(\mathcal{T} / 2)=a_{1}$. This corresponds in the $\left(\varphi_{1}, \varphi_{2}\right)$-plane to the point $\left(0, \frac{3}{4}-p^{2}\right)$. This point is the intersection between the quadric $\left(w_{3}=0\right)$ with the $\varphi_{2}$-axis. In this case the orbit is closed.

End of the proof of Theorems 1.1, 1.2 and 3.1. Theorem 3.1 follows directly from Proposition 3.1. Let $\left(\varphi_{1}, \varphi_{2}\right)$ be the periodic solution of (7)-(8) satisfying (9) with $\varphi_{2}(0)=p=\sqrt{3 / 8}$, and let $a$ be the period of this solution. 
From Proposition 2.1, the Riemannian metric $\left(\varphi_{1}^{2}(y)+\varphi_{2}^{2}(y)\right) g_{a}$ is, up to a dilatation, the only extremal metric of the functional $\lambda_{1}$ under area preserving deformations. This proves Theorem 1.1. Proposition 2.1 also tells us that this metric is the only one to admit a homothetic minimal immersion into a sphere by the first eigenfunctions. Moreover, such an immersion is unique up to isometries and is given by

$$
\rho \circ h=\left(\sqrt{1-\varphi_{1}^{2}(y)-\varphi_{2}^{2}(y)}, \varphi_{1}(y) e^{i x}, \varphi_{2}(y) e^{2 i x}\right)
$$

for some $\rho \in O(5)$ (see Remark 2.1). This proves Theorem 1.2.

\section{REFERENCES}

[1] Ankiewicz A. and Pask C., The complete Whittaker theorem for two-dimensional integrable systems and its application, J. Phys. A: Math. Gen. 16 (1983) 4203-4208.

[2] Arnold V. I., Mathematical methods of classical mechanics (second edition), SpringerVerlag (1989).

[3] Bavard C., Inégalité isosystolique pour la bouteille de Klein, Math. Ann. 274 (1986) 439-441.

[4] Bourguignon J-P., Li P. and Yau S.T., Upper bound for the first eigenvalue of algebraic submanifolds, Comm. Math. Helv. 69 (1994) 199-207.

[5] Colbois B. and Dodziuk J., Riemannian metrics with large $\lambda_{1}$, Proc. Am. Math. Soc. 122, (1994) 905-906.

[6] Colbois B. and El Soufi A., Extremal eigenvalues of the Laplacian in a conformal class of metrics: The conformal spectrum, Annals of Global Analysis and Geometry 24, (2003) 337-349.

[7] El Soufi A. and Ilias S., Le volume conforme et ses applications d'après Li et Yau, Sém. Théorie Spectrale et Géométrie, Institut Fourier, année 1983-1984, No.VII, (1984).

[8] El Soufi A. and Ilias S., Immersions minimales, première valeur propre du laplacien et volume conforme, Math. Ann. 275 (1986) 257-267.

[9] El Soufi A. and Ilias S., Riemannian manifolds admitting isometric immersions by their first eigenfunctions, Pac. J. Math. 195, (2000) 91-99 .

[10] EL Soufi A. and Ilias S., Addendum to "Riemannian manifolds admitting isometric immersions by their first eigenfunctions", preprint University of Tours.

[11] El Soufi A. and Ilias S., Extremal metrics for the $\lambda_{1}$ functional in a conformal class, Proc. Am. Math. Soc. 131, (2003) 1611-1618.

[12] Ferus D. and Pedit F., $S^{1}$-equivariant minimal tori in $S^{4}$ and $S^{1}$-equivariant Willmore tori in $S^{3}$, Math. Z. 204, no. 2, (1990) 269-282.

[13] Girouard A., Valeur propre fondamentale et divergence conforme sur le tore, Preprint, arxiv: math.SP/0510279.

[14] Hersch J., Quatre propriétés isopérimétriques des membranes sphériques homogènes, C. R. Acad. Sc. Paris, Série A 270, (1970) 1645-1648.

[15] Jakobson D., Nadirashvili N. and Polterovich I., Extremal metric for the first eigenvalue on a Klein bottle, arXiv: math.SP/0311484 26 Nov 2003, to appear in Canadian Journal of Mathematics.

[16] Li P. and Yau S.T., A new conformal invariant and its applications to the Willmore conjecture and the first eigenvalue of compact surfaces, Invent. Math. 69 (1982) 269-291.

[17] Montiel S. and Ros A., Minimal immersions of surfaces by the first eigenfunctions and conformal area. Invent. Math., 83, (1985) 153-166.

[18] Nadirashvili N., Berger's isoperimetric problem and minimal immersions of surfaces, GAFA 6 (1996) 877-897.

[19] Polterovich L., Symplectic aspects of the first eigenvalue, J. Reine Angew. Math. 502, (1998) 1-17.

[20] Takahashi T., Minimal immersions of Riemannian manifolds, J. Math. Soc. Jap. 18, (1966) 380-385. 
[21] E.T. Whittaker, A Treatise on the Analytical Dynamics of Particles and Rigid Bodies, Cambridge University Press, London, 1937.

[22] Yang P. and Yau S.T., Eigenvalues of the Laplacian of compact Riemann surfaces and minimal submanifolds, Ann. Scuola Norm. Sup. Pisa 7, (1980) 55-63.

Ahmad El Soufi and Hector Giacomini: Université François Rabelais de Tours, Laboratoire de Mathématiques et Physique Théorique, UMR-CNRS 6083, Parc de Grandmont, 37200 Tours, France.

Mustapha Jazar: Lebanese University, Mathematics Department, P.O. Box 155-012, BEIRUT, LEBANON.

E-mail address: ahmad.elsoufi@univ-tours.fr, hector.giacomini@univ-tours.fr, mjazar@ul.edu.lb 